\title{
A Prospective Study on Effect of Epidural Blood Patch in Treating Postdural Puncture Headache
}

\author{
Authors \\ Dr Ananta Venkata Raman ${ }^{1}$, Dr Terupalli Varaprasada Rao ${ }^{2}$ \\ ${ }^{1}$ Associate Professor, Department of Anaesthesia, Malla Reddy Institute of Medical Sciences, India \\ ${ }^{2}$ Assistant Professor, Department of Anaesthesia, Malla Reddy Institute of Medical Sciences, India \\ *Corresponding Author \\ Dr Terupalli Varaprasada Rao \\ H.no: c-108, Phase-2, Hill colony, Vanasthalipuram, Hyderabad-500070, India
}

Email:avis.reddy@gmail.com

\begin{abstract}
Epidural blood patch (EBP) is most common treatment of post-dural puncture headache, but its mode of action and effectiveness remain a matter of debate. Now a days Spinal anaesthesia is used widely. This dural puncture is associated with CSF leak which causes headache because of decreased intracranial pressure. The aim of this study was to assess both the effectiveness of epidural blood patch in treating postdural puncture headache and the predictive factors of failure of EBP on severe post-dural puncture headache. In this prospective study a total of 180 patients were involved. 180 patients received epidural blood patch for postdural puncture headache The EBP effect was classified into complete relief (disappearance of all symptoms),incomplete relief of symptoms (clinically improved patients who recovered sufficiently to perform normal daily activity), and failure (persistence of severe symptoms). The frequency rates of complete relief, incomplete relief of symptoms, and failure after EBP were 79.42\%, $8.82 \%$ and $11.76 \%$ respectively. To conclude Epidural blood patch is an effective treatment of severe post-dural puncture headache. In the present study it showed a success rate of $88.24 \%$ after receiving first EBP.

Keywords: Epidural blood patch, dural puncture, headache, spinal anaesthesia.
\end{abstract}

\section{Introduction}

Headache and backache are the most common symptoms associated with dural puncture. ${ }^{[1]}$ Wynter and Quincke used aspiration of CSF as a mode of treatment of raised intracranial pressure, and introduced spinal anaesthesia in the year $1891^{[2]}$. In 1898 the incidence of postdural puncture headache was around $66 \%$ and the incidence decreased to $11 \%$ with the advent of
$22 \mathrm{G}$ to $29 \mathrm{G}$ spinal needles. Gormley in 1960 suggested the injection of autologous blood in the lumbar epidural space ${ }^{[3]}$ and later Di Giovanni and Dunbar introduced it. ${ }^{[4]}$ Many studies have been done on epidural blood patch to evaluate its effectiveness.

Significant morbidity was caused amongst patients by the post dural puncture headache. Various vestibular, neck and ocular symptoms are 
associated with postdural puncture headache and these are the clinical signs act as a diagnostic criteria for detecting accidental puncture. ${ }^{[5]}$ Spontaneous evolution of post dural puncture headache (PDPH) results mainly in recovery within a few days, but it has been observed that many cases are very incapacitating and may persist for several months. Severe and even fatal complications have been observed. ${ }^{[6]}$

The aim of this prospective observational study was to assess both the effectiveness and the predictive factors of failure of EBP on severe post dural puncture headache (PDPH). There are two theories which have been proposed to explain EBP efficiency in PDPH. ${ }^{[7]}$ The first theory suggests that the injected blood creates a clot which is particularly adherent to the dura mater, preventing CSF leak through directly patching the hole in the dura. ${ }^{[8]}$ The second theory suggests that the volume of blood injected in the epidural space increases CSF pressure and thus reducing traction on brain and meningeal structures which is causing relief of symptoms. ${ }^{[9]}$ Both these two theories are probably correct. The first theory probably explains the longlasting effectiveness of the EBP, whereas the second theory probably explains the rapid onset of symptom relief after EBP.

\section{Materials and Methods}

Patients who were admitted in our hospital between December 2014 and October 2016 were included in this study. In this prospective study a total of 180 patients were involved. 180 patients received epidural blood patch for postdural puncture headache The EBP effect was classified into complete relief (disappearance of all symptoms), incomplete relief of symptoms (clinically improved patients who recovered sufficiently to perform normal daily activity), and failure (persistence of severe symptoms).

All patients were treated in our hospital with EBP for PDPH were included in our prospective observational study. Diagnosis criteria of severe PDPH were a clinical history of dural puncture associated with severe postural symptoms in patients who were disabled in their daily activities and needed to stay in bed for a part of the day. Patients with hemolysis and suspected infection was not included in the study. Severely immunocompromised patients were also excluded Under strict surgical aseptic conditions, Epidural blood patch was conducted by experienced staff anesthesiologists, on a patient in a sitting position with the legs dependent. Using a 17-gauge or 18gauge Tuohy needle, the lumbar epidural space was located by the loss-of-resistance technique with fluid. the level of EBP was chosen by the operator. When the epidural space had been localized, using strict aseptic technique an autologous venous blood sample was drawn (by a second operator) from an antecubital vein into a plastic syringe. The drawn blood was slowly injected into the epidural space at a speed of approximately $0.3 \mathrm{ml} / \mathrm{s}$ through the Tuohy needle. The injection was always stopped $2 \mathrm{ml}$ after the appearance of pain in the back, buttocks, or legs. In absence of any pain, the injected volume chosen by the operator was at least $20 \mathrm{ml}$. The patient was asked to stay lying for one hour in dorsal decubitus position and the cannula was then removed, The effectiveness of EBP was evaluated after one hour by asking the patient to stand up and walk. Treatment effectiveness was again assessed at day one by the attending physician and at day 15 by a telephone interview with the patient, The results of the EBP treatment on clinical signs were noted according to the classification.

\section{Results}

In two year prospective observational study 180 patients were included in this study. Out of these 180 patients 135 were females and 45 were male patients. In 34 patients dural puncture lead to post dural puncture headache, out of these 34,14 patients were of spinal anaesthesia and 20 patients were of epidural anaesthesia.

CSF leakage Symptoms were noted after a delay of 1-9 days after dural puncture. Majority of the 
patients presented with headache (97\%), with neck pain $(81 \%)$, Vestibular signs like nausea and vomiting were present in $70 \%$ of patients. ocular signs were present in $36 \%$ cases and Cochlear signs were present in $36 \%$ cases.

Out of 34 patients who received EBP for PDPH, 27 patients $(79.42 \%)$ showed complete relief of symptoms, three patients (8.82\%) showed incomplete relief of symptoms and four patients $(11.76 \%)$ showed persistence of symptoms. These four patients again received a second EBP after 5 days, out of these four patients only one was considered as treatment failure, remaining three patients has complete relief of symptoms. Fever without neurologic complication was the only complication encountered during this observantional study which also resolved spontaneously.

\section{Discussion}

The most frequently reported symptoms are Headache and neck symptoms. According to our prospective observational study, headache was the most common symptom and similar to a study conducted by Stride and Croper the incidence of dural puncture was greater with larger gauge needle. ${ }^{[10]}$ According to a study by Baysinger et $\mathrm{al}^{[11]}$ in 2011, In North America many anaesthetics still resort to older treatment of oral caffeine, NSAIDS and bed rest as a treatment for postdural puncture heachache. In the study by Bradbury et al and Norris et $\mathrm{al}^{[12,13]}$, lateral orientation of the needle decreased the incidence of postdural puncture headache from $2.4 \%-1.4 \%$.

In the present study the effectiveness of a first EBP to treat PDPH was high, when both complete and incomplete relief of symptoms (93\%) were considered as a success, as previously reported studies. ${ }^{[14]}$ When only complete relief was considered, the success rate of the EBP was of $79.42 \%$ in our present study. In the literature the reported EBP effectiveness remains highly variable because some investigators in their studies consider only total relief from symptoms as success, whereas others investigators included incomplete relief of symptoms as success.
In review of literature, Vercauteren et $\mathrm{al}^{[15]}$, study says that the effectiveness of EBP is highly variable as the criteria of success are different amongst different studies. In present study Headache and neck symptoms are the most frequently reported symptoms and the symptom of CSF leak appear after a delay of approximately one day. In 2011, According to Baysinger et $\mathrm{al}^{[11]}$, many anaesthetics in North America Anaesthesiologists are still avoiding newer techniques like epidural blood patch, intravenous cosyntroprin and neuraxial morphine and still resort to older treatment of NSAIDS, oral caffeine and bed rest as a treatment for postdural puncture heachache. According to our study EBP is safe and effective treatment.

Banks et al in their study showed that complete relief was seen in $50 \%$ patients, $38 \%$ patients got partial relief and $12 \%$ had no relief in symptoms on receiving epidural blood patch. In present prospective observational study who received EBP for PDPH, 79.42\% showed complete relief of symptoms, $8.82 \%$ showed incomplete relief of symptoms and $11.76 \%$ showed persistence of symptoms.

\section{Conclusion}

To conclude epidural blood patch is an effective and safe treatment, around $79 \%$ of the patients had complete relief from symptoms. Epidural blood patch is an effective treatment of severe postdural puncture headache. In the present study it showed a success rate of $88.24 \%$ after receiving first EBP.

\section{References}

1. Reynolds F. Dural puncture and headache. Br Med J. 1993; 306:874-6.

2. Pearce JM. Walter Essex Wynter, Quincke, and lumbar puncture. J Neurol Neurosurg Psychiatry. 1994;57:179.

3. Gormley JB: Treatment of postspinal headache. ANESTHESIOLOGY 1960; 21:565-6. 
4. Di Giovanni AJ, Dunbar BS: Epidural injections of autologous blood for postlumbar-puncture headache. Anesth Analg 1970; 49:268-71.

5. Halpern S, Preston R: Postdural puncture headache and spinal needle design: Metaanalyses. Anesthesiology. 1994; 81:1376-83.

6. Gielen M: Post dural puncture headache (PDPH): A review. Reg Anesth 1989; 14:101-6.

7. Beards SC, Jackson A, Griffiths AG, Horsman EL: Magnetic resonance imaging of extradural blood patches: Appearances from $30 \mathrm{~min}$ to $18 \mathrm{~h}$. Br J Anaesth 1993; $71: 182$.

8. Djurhuus $\mathrm{H}$, Rasmussen $\mathrm{M}$, Jensen $\mathrm{EH}$ : Epidural blood patch illustrated by CTepidurography. Acta Anaesthesiol Scand 1995; 39:613-7

9. Colonna-Romano P, Linton P: Cervical dural puncture and lumbar extradural blood patch. Can J Anaesth 1995; 42:1143-4

10. Stride PC, Cooper GM: Dural taps revisited: A 20-year survey from Birmingham Maternity Hospital. Anaesthesia. 1993;48:247-55.

11. Baysinger, C.L., Pope, J.E., Lockhart, E.M. and Mercaldo, N.D. The Management of Accidental Dural Puncture and Postdural Puncture Headache: A North American Survey. Journal of Clinical Anesthesia. 2011;23:349-360.

12. Bradbury, C.L., Singh, S.I., Badder, S.R., Wakely, L.J. and Jones, P.M. Prevention of Post-Dural Puncture Headache in Parturients: A Systemic Review and MetaAnalysis. Acta Anaesthesiologica Scandinavica. 2013;57: 417-430.

13. Norris, M.C., Leighton, B.L. and DeSimone, C.A. Needle Bevel Direction and Headache after Inadvertent Dural Puncture. Anesthesiology. 1989;70:729731.
14. Abouleish E, de la Vega S, Blendinger I, Tio TO: Long-term follow-up of epidural blood patch. Anesth Analg 1975; 54:45963.

15. Vercauteren MP, Hoffmann VH, Mertens E, Sermeus L, Adriaensen HA: Seven-year review of requests for epidural blood patches for headache after dural puncture: Referral patterns and the effectiveness of blood patches. Eur J Anaesthesiol. 1999;16:298-303. 